\title{
Evaluation of Changes in Actin Filaments of RK13 Cells Infected with Malassezia pachydermatis
}

\author{
Iris del Socorro Flores Rodríguez¹, Tonatiuh Alejandro Cruz Sánchez¹, José Luis Nieto Bordes², \\ Francisco Rodolfo González Díaz², Carlos Ignacio Soto Zárate², \\ Carlos Gerardo García Tovar2*
}

\footnotetext{
${ }^{1}$ Laboratorio 6 de Bioprospección Microbiológica, Unidad de Investigación Multidisciplinaria (UIM), Facultad de Estudios Superiores Cuautitlán, UNAM, Cuautitlán Izcallí, México ${ }^{2}$ Laboratorio 4 de Morfología Veterinaria y Biología Celular, Unidad de Investigación Multidisciplinaria (UIM), Facultad de Estudios Superiores Cuautitlán, UNAM, Cuautitlán Izcallí, México Email: *cgarciatov@yahoo.com.mx
}

How to cite this paper: Flores I., Cruz, T.A., Nieto, J.L., González, F.R., Soto, C.I. and García, C.G. (2018) Evaluation of Changes in Actin Filaments of RK13 Cells Infected with Malassezia pachydermatis. Open Journal of Veterinary Medicine, 8, 15-24.

https://doi.org/10.4236/ojvm.2018.82003

Received: December 14, 2017

Accepted: February 25, 2018

Published: February 28, 2018

Copyright $\odot 2018$ by authors and Scientific Research Publishing Inc. This work is licensed under the Creative Commons Attribution International License (CC BY 4.0).

http://creativecommons.org/licenses/by/4.0/

(c) (i) Open Access

\begin{abstract}
Background: Malassezia pachydermatis is the main causative agent of canine otitis and also of a myriad of dermatological problems in companion animals; its interaction mechanisms with host cells are still unclear. Objectives: To establish an in vitro infection model of $M$. pachydermatis-exposed RK13 cells in order to evaluate cell morphological changes as well as changes in the structure of actin filaments. Methods. Cultures of RK13 cells were infected with $M$. pachydermatis, alterations caused by the yeast were evaluated by optical and fluorescence microscopy. Results: $M$. pachydermatis adheres itself to the cell and produces the formation of multiple agglomerates that cause changes in cell morphology, formation of cell aggregates in overlays, presence of syncytia and destruction of cell culture structure. The damaged cells presented changes in the actin filaments consisting of thickening of the cell cortex and loss of stress fibers. On the other hand, the formation of perinuclear actin rings in the yeasts was observed. Conclusions: An in vitro infection model was established with $M$. pachydermatis and alterations in cell morphology were observed consisting of changes in the structure of the actin filaments, overgrowth of the cells and the presence of syncytia.
\end{abstract}

\section{Keywords}

Malassezia pachydermatis, RK13 Cells, Actin Filaments 


\section{Introduction}

Malassezia pachydermatis is a yeast of great importance in veterinary medicine, it is part of the skin's normal microbiota of many animals and it is associated with changes in the immunological mechanisms of its host; the proliferation of this yeast can result in the clinical presentation of otitis, seborrhea, atopy and allergic dermatitis [1]. The external acoustic meatus represents a fragile environment in which inflammatory changes are capable of causing alterations in the skin's microbiota such that the uncontrolled proliferation of M. pachydermatis becomes a perpetuating factor of canine external otitis. By itself, this yeast is responsible for about 30 to $80 \%$ of canine otitis externa cases and is also associated with about $30 \%$ of seborrheic dermatitis and atopy cases in dogs [2] [3]. In humans, $M$. pachydermatis can cause fungaemias, especially in newborns that are kept in intensive therapy [4] and in immunocompromised patients; moreover, it has been observed that it can even cause alterations in immunocompetent patients, thus reveling its zoonotic potential [5].

In spite of being one of the main causative agents responsible for a number of cutaneous problems in animals, many of its pathogenic mechanisms are still poorly understood, especially those relating to its normal presence as part of the skin's microbiota, which creates immunological tolerance.

Recent studies have mainly focused on determining the events of the interactions between pathogens and host cells that give rise to colonization and the creation of a specialized niche that ensures pathogen survival [6] [7]. A common target of these studies is the host cell's cytoskeleton, which is utilized by different pathogens (virus, bacteria and fungi) for several purposes, including attachment, internalization, movement between and inside cells, vacuole formation, remodeling and avoidance of phagocytosis [8] [9].

Actin filaments are a part of the cytoskeleton that are related to the maintenance and changes in cell shape and movement by means of structures that are formed by the rearrangements of the filaments induced as a response to diverse intra and extracellular signals. In mammal cells these filaments form bundles and networks that give rise to structures such as the cellular cortex, stress fibers and lamellipodia [10] [11] [12]. In yeasts they form patches and cables [13].

Little is known about the pathogenic mechanisms that this yeast exerts on the tissues it affects. During colonization and diffusion of the pathogen, a key role is played by the changes induced in actin filament organization, which is a mechanism that has already been demonstrated by other yeast such as Sporothrix schenkii, Candida albicans, Candida glabrata y Malassezia furfur, therefore, it is possible that Malassezia pachydermatis also exhibits such mechanism [9] [14] [15] [16].

The objective of the present study was to establish an in vitro model of RK13-cell infection with $M$. pachydermatis and to evaluate the capacity of this yeast to colonize the culture, as well as the alterations in cell morphology and in the actin filaments. 


\section{Materials and Methods}

\subsection{M. pachydermatis Culture and Growth Conditions}

M. pachydermatis (reference strain ATCC-14522), generously donated by Dr. Tonatiuh Cruz from the laboratory 4 of the Multidisciplinary Research Unit, UNAM, Cuautitlán was cultured for 72 hours at $33^{\circ} \mathrm{C}$ in Sabouraud dextrose agar (BD Bioxon), containing $1 \%$ casein and $4 \%$ dextrose. Yeasts were recollected from solid medium, washed three times with PBS and resuspended in SDA liquid medium.

\subsection{RK13 Cell Culture}

RK13 cells (ATCC CCL-37) were cultured in DMEM medium supplemented with $10 \%$ Fetal Bovine Serum (FBS) and were kept at $37^{\circ} \mathrm{C}$ in an atmosphere with $5 \%$ $\mathrm{CO}_{2}$ and $95 \%$ humidity.

\subsection{RK13 Cell Infection with M. pachydermatis}

Cells were seeded in 24-well culture plates that contained previously sterilized rounded coverslips. Cellular cultures were divided into three groups: Group I, uninfected control culture; Group II, cells cultured in SDA medium only, and group III, cellular culture infected with $M$. pachydermatis. Cells were infected at a ratio of 10:1 $\left(1 \times 10^{6}\right.$ yeasts). Each assay was performed three times and each time was monitored at 24,48 and 72 hours post incubation at $37^{\circ} \mathrm{C}$.

\subsection{Evaluation of Adherence Capacity and Damage to the Cellular Monolayer}

Evaluation of $M$. pachydermatis-infected RK13 cells was realized by optical microscopy with the intention of determining the presence of morphological changes in the cellular cultures, as well as the formation of yeast colonies. In order to do this, coverslips containing the cultures from each of the three groups were taken from the wells at 24, 48 and 72 hours and washed with PBS in order to remove any non-adhered yeasts. After this, all cultures were fixed with $10 \%$ aqueous formalin in PBS, mounted on microscope slides and observed.

\subsection{Evaluation of the Changes Induced on the Actin Filaments}

Interaction between the yeast and culture cells was evaluated by double-staining direct fluorescence. Coverslips with RK13 culture cells from control and infected groups were removed from their medium, fixed with $10 \%$ aqueous formalin in PBS during 20 minutes, permeated with $0.5 \%$ Triton X-100 in PBS for 5 minutes, and to help reduce nonspecific binding the cells were incubated with $1 \%$ BSA in PBS during 20 minutes. For detection of the actin filaments, we use Tetramethyl Rhodamine-IsoThioCyanate (TRITC) labeled Phalloidin (Sigma-Aldrich ${ }^{\circ}$ ), diluted 1:100 in PBS during $20 \mathrm{~min}$ in a humid chamber under conditions of darkness. Finally, the coverslips were mounted on slides using mounting medium with 4'6-diamino-2-phenylindole (DAPI), to detect the nuclei (Ultracruz ${ }^{\circledR}$ 
Mounting Medium for Fluorescence, Santa Cruz, CA, USA).

\subsection{Fluorescence Microscopy}

In order to evaluate the changes induced by the yeast on the actin cytoskeleton, observation by fluorescence microscopy was performed (Zeiss Axioscop 40, coupled to an Evolution VF Cooled Color camera from Media cybernetics). Alterations encountered in cellular cultures and actin filaments were also analyzed by confocal microscopy (Nikon A1R STORM confocal microscope).

\section{Results}

Optic microscopy revealed that uninfected cells formed monolayers with normal structure and normal cellular morphology (Figure 1(a)), the same as the cells in SDA medium (data not shown). In contrast, yeast-infected cells displayed numerous changes in the structure of the monolayer as well as alterations in cellular morphology such as rounded cells and loss of the monolayer integrity. Formation of yeast colonies in grape-like clusters was also observed (Figure 1(c)).

Results from fluorescence microscopy revealed that infection by $M$. pachydermatis induces changes in the actin cytoskeleton. Uninfected cells (groups I and II) displayed a normal structure of the actin cytoskeleton (Figure 1(b)). Infected cells exhibited loss of cellular morphology on the yeast attachment sites to the cell, apparently overgrown cellular clusters in circular arrangements were formed, cells were elongated and rounded with loss of stress fibers and thickened cellular cortex (Figure 1(d)). When performing the analysis of the cultures in which actin filaments and nuclei were detected by the direct fluorescence assay, autofluorescence was observed in the yeasts.

An infected culture was examined by confocal microscopy, observing the formation of syncytia and small circular actin structures that corresponded to the yeast's attachment sites. These circular structures collocate with yeast's nuclei position when merge is performed, each nucleus is surrounded by actin filaments ring (Figure 2).

In order to demonstrate that $M$. pachydermatis was stimulating cellular culture overgrowth beyond that of a monolayer, uninfected and infected cellular cultures were analyzed by confocal microscopy, orthogonal view Z-stack. In Figure 3 we show uninfected and infected cellular cultures that correspond to the images obtained after they were merged.

Uninfected cellular cultures in the XY plane can be observed in Figure 3(a) and Figure 3(c), here we can appreciate the formation of an unaltered cellular monolayer, with normal cellular morphology and actin filaments with no structural changes. In Figure 3(b) an infected cellular culture is shown, here we can observe grape-like clusters of yeasts in their sites of attachment to the cells. Overgrown cellular aggregates with loss of normal morphology are also noticed (Figure 3(d)). In Figure 3(c) and Figure 3(d) orthogonal Z-stack views are shown, they further demonstrate the overgrowth of the monolayer. Uninfected 
Optic microscopy
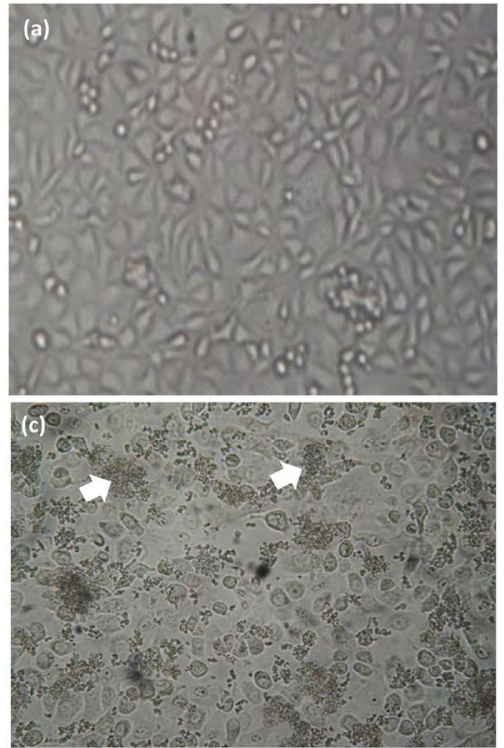

Fluorescence microscopy
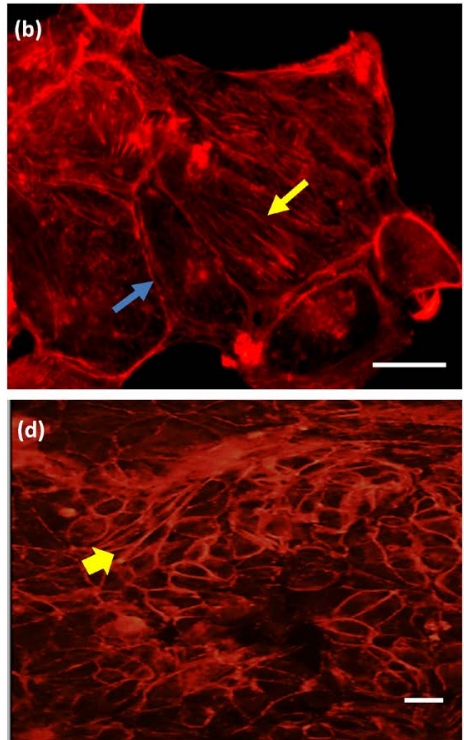

Figure 1. RK13 cells infected with $M$. pachydermatis. (a) and (b): Uninfected control group; (c) and (d): M. pachydermatis-infected cellular culture. (b) and (d): Actin filaments detected with TRITC labeled phalloidine (direct fluorescence). (b): Cellular cortex (blue arrow), stress fibers (yellow arrow); (c): M. pachydermatis colonies (white arrow); (d): Circular cellular aggregates on the sites of yeast attachment (yellow arrow). (a), (c) and (d) $(40 \times)$; b $(100 \times)$. Barr $=10 \mu \mathrm{m}$.
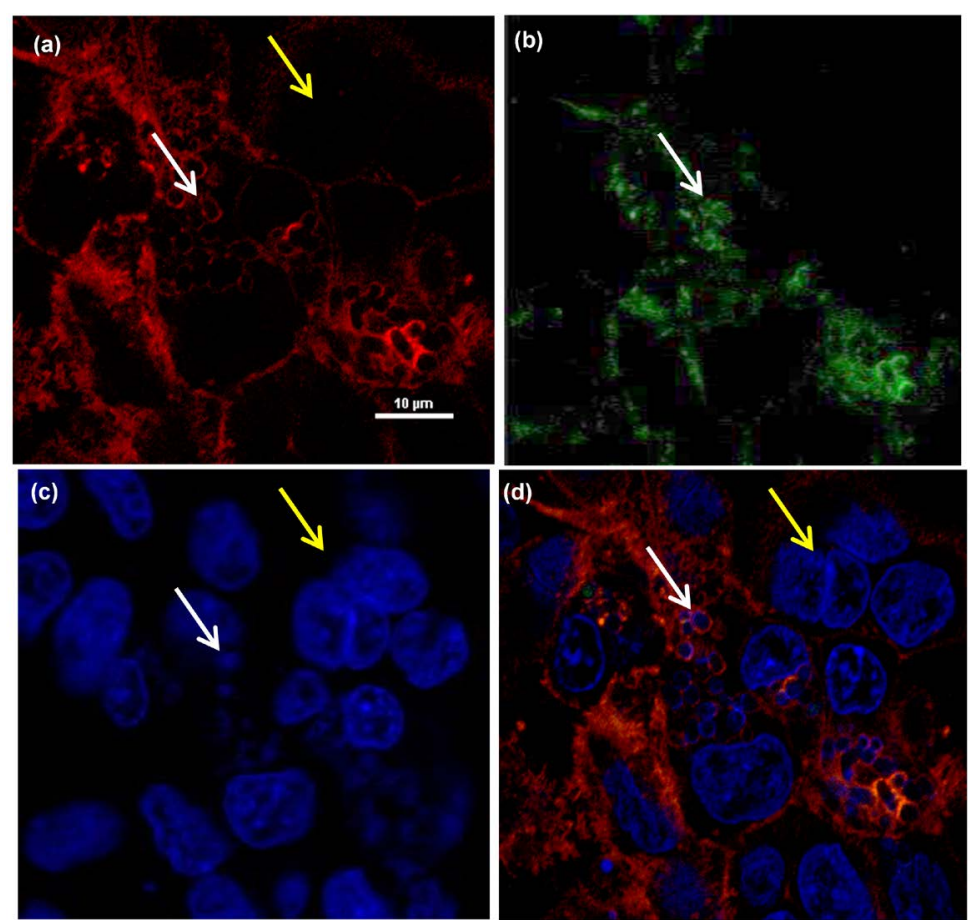

Figure 2. RK13 cells infected with $M$. pachydermatis showing syncytia formation and presence of yeast's perinuclear rings. (a) Actin filaments detected with TRITC labeled phalloidine; (b) M. pachydermatis; (c) Nuclei detected with DAPI; (d) Merge. Syncytia formation (yellow arrow) and yeast's perinuclear rings (white arrow). Confocal microscopy $(60 \times)$. Barr $=10 \mu \mathrm{m}$. 

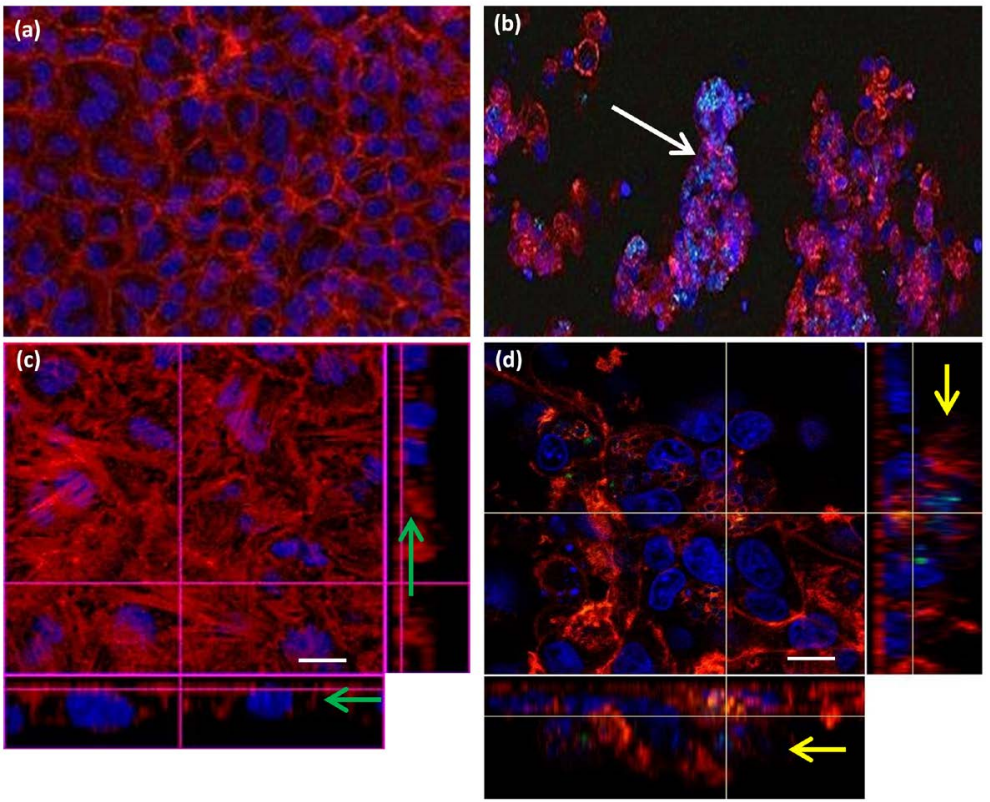

Figure 3. RK13 cells displaying overgrowth of the cellular monolayer during infection by M. pachydermatis. Direct fluorescence; actin filaments detected with TRITC labeled phalloidine, nuclei detected with DAPI and autofluorescent $M$. pachydermatis using a green wavelength. (a) and (c) Uninfected RK13 cells; (b) and (d) RK13 cells infected with M. pachydermatis. (a) and (b) correspond to the images obtained in the XY plane; (c) and (d), lateral and inferior boxes correspond to the orthogonal view Z-stack. B: Yeast colony (white arrow). (c) Orthogonal view Z-stack showing a $16 \mu \mathrm{m}$ thick monolayer (green arrow); (d) Orthogonal view Z-stack showing an overgrown monolayer with a $32 \mu \mathrm{m}$ thickness (yellow arrow). Confocal microscopy. Barr $=10 \mu \mathrm{m}$.

cellular cultures have a thickness of $16 \mu \mathrm{m}$ and the presence of a single monolayer is clearly appreciated by the observation of all nuclei in a single plane (Figure 3(c)), in contrast, a thickened cellular layer of $32 \mu \mathrm{m}$ can be observed in infected cultures, which correlates with a cellular overgrowth of several layers, this can also be easily noted by the observation of the nuclei in different strata (Figure 3(d)).

\section{Discussion}

In the last few years there has been an increasing interest in the study of the ability of the pathogens to invade and reproduce in non-phagocytic cells in order to ensure their survival. Yeasts such as Candida albicans, C. glabrata and Malassezia furfur are associated with chronic infections that are difficult to eliminate and their internalization in non-phagocytic cells by modulation of the actin cytoskeleton has been reported [14] [15] [16] [17]. However, M. pachydermatis capacity to invade epithelial cells and infect them through alterations of the actin filaments has not been studied.

The present study provides evidence that helps in the understanding of the interactions between a fungal pathogen and epithelial cells following an in vitro model. Our results show that $M$. pachydermatis induces alterations of the epi- 
thelial cell monolayer's structure, causing changes in cell morphology and overgrowth of the cells, these extra-layers present changes in morphology and alterations in their actin filaments, as evidenced by the loss of stress fibers; syncytia formation was also observed. All of the previously mentioned alterations point to the fact that the cells are undergoing changes that are being induced by the yeasts.

In a previous work [17], it was pointed that the proliferation rate was similar for infected cells and those belonging to the control group, nonetheless, we observed an increase in cellular proliferation on the attachment sites of the yeast. According to that observed by confocal microscopy, such cellular aggregates supporting the theory that $M$. pachydermatis in vivo causes hyperkeratosis and hyperplasia, both of which are evident as macroscopic and histologic lesions [18] [19] and, hypothetically, the outcome of an inflammatory and/ or an hypersensitivity reaction to the substances secreted by the yeasts and its antigens [20] [21] [22], such as: alkaline and acid phosphatase, sulfatase chondroitin, esterase, galactosidase, glucosidase, hialuronidase, lipase, lecithinase, peroxidase, protease and urease, among others that have been the target of great interest and may be pathogenically involved [23] [24]. The damage caused by the presence of the yeast, according to our observations, could relate to the release of such enzymes instead of being a consequence of an immune reaction, given the fact that during the course of this study only an infected culture was used and cellular proliferation was observed in the attachment sites between the yeasts and cells, which in turn suggest that changes are induced in order to act as means by which the yeast protects itself and avoids immune responses. These findings could help to understand the pathogenic mechanism of this yeast and to explain the presence of epidermic hyperkeratosis observed in biopsies obtained from infected tissues, as well as the difficulties encountered during the therapeutic management of otitis and dermatitis caused by $M$. pachydermatis.

Small circular actin structures limiting the nuclei of the yeasts were also observed, such structures could correlate to the presence of actin rings that have been previously reported in Fellomyces fuzhouensis [25] and Cryptococcus neoformans [26].

\section{Conclusion}

In conclusion, we were able to establish an infection model of RK13 cells by $M$. pachydermatis. Yeasts adhered themselves to culture cells forming grape-like colonies. The more relevant findings were: alterations in cellular morphology and in the structure of the actin filaments; monolayer overgrowth in the sites of yeast attachment and syncytia formation. Also of relevance, is the fact that, to our knowledge, this constitutes the first study to report the presence of perinuclear rings in $M$. pachydermatis. These findings will help to further clarify the pathogenic mechanism of this yeast and to explain the presence of epidermic hyperkeratosis observed in biopsies obtained from infected tissues as well as the 
difficulties encountered during the therapeutic management of otitis and dermatitis caused by $M$. pachydermatis.

\section{Acknowledgements}

We are grateful to the Consejo Nacional de Ciencia y Tecnología (CONACYT) México for grant \# 71312, afforded for the carrying out of Master in Science studies, as well as for projects PAPIIT IT 200915 (DGAPA-UNAM), PIAPI 1632 and PIAPI 1602 (FES-Cuautitlán UNAM).

\section{Conflict of Interest}

The authors declare that this article has not been send to nor published by other journals and that no conflict of interest exists.

\section{References}

[1] Hnilica, K.A. and Patterson, A.P. (2017) Small Animal Dermatology. 4th Edition, Elsevier, Amsterdam.

[2] Kumar, A., Singh, K. and Sharma, A. (2002) Prevalence of Malassezia pachydermatis and Other Organisms in Healthy and Infected Dog Ears. Israel Journal of Veterinary Medicine, 57, 145-148.

http://www.ibrarian.net/navon/paper/PREVALENCE_OF_MALASSEZIA_PACHY DERMATIS_AND_OTHER_.pdf?paperid=19497534

[3] Nowakiewics, A. and Ziolkowska, G. (2013) Comparative Analysis of Protein Profiles of Malassezia pachydermatis Strains Isolated from Healthy Dogs and Dogs with Otitis Externa. Bulletin of the Veterinary Institute in Pulawy, 57, 41-46. https://www.degruyter.com/downloadpdf/j/bvip.2013.57.issue-1/bvip-2013-0008/bv ip-2013-0008.pdf

[4] Al-Sweih, N., Ahmad, S., Josep, L., Khan, S. and Khan, Z. (2014) Malassezia pachydermatis Fungemia in a Preterm Neonate Resistant to Fluconazole and Flucytosine. Medical Mycology Case Reports, 5, 9-11. https://doi.org/10.1016/j.mmcr.2014.04.004

[5] Fan, Y.M., Huang, W.M., Li, S.F., Wu, G.F., Lai, K. and Chen, R.Y. (2006) Granulomatous Skin Infection Caused by Malassezia pachydermatis in a Dog Owner. Archives of Dermatology, 142, 1181-1184. https://doi.org/10.1001/archderm.142.9.1181

[6] Gouin, E., Welch, M.D. and Cossart, P. (2005) Actin-Based Motility of Intracellular Pathogens. Curr Op Microbiol, 8, 35-45. https://doi.org/10.1016/j.mib.2004.12.013

[7] Vallace, B.A. and Finlay, B.B. (2000) Exploration of Host Cells by Enteropathogenic Escherichia coli. Proceedings of the National Academy of Sciences of the United States of America, 97, 8799-8806. https://doi.org/10.1073/pnas.97.16.8799

[8] Gruenheid, S. and Finlay, B.B. (2003) Microbial Pathogenesis and Cytoskeletal Function. Nature, 422, 775-781. https://doi.org/10.1038/nature01603

[9] Sabanero, L.M., Tsutsumi, F.V., Barbosa, S.G., López, R.E. and Sandoval, B.G. (2006) Interaction of Yeasts of Sporothrix schenckii with Epithelia. Research and Science, 36, 10-14. http://www.uaa.mx/investigacion/revista/archivo/revista36/REVISTA\%2036.pdf

[10] Alberts, B., Johnson, A., Lewis, J., Morgan, D., Raff, M., Roberts, K. and Walter, P. (2015) Molecular Biology of the Cell. 6th Edition, Garland Science, New York. 
[11] Roubinet, C., Tran, P.T. and Piel, M. (2012) Common Mechanisms Regulating Cell Cortex Properties during Cell Division and Cell Migration. Cytoskeleton, 69, 957-972. https://doi.org/10.1002/cm.21086

[12] Tojkander, S., Gateva, G. and Lappalainen, P. (2012) Actin Stress Fibers-Assembly, Dynamics and Biological Roles. Journal of Cell Science, 125, 1855-1864. https://doi.org/10.1242/jcs.098087

[13] Moseley, J.B. and Goode, B.L. (2006) The Yeast Actin Cytoskeleton: From Cellular Function to Biochemical Mechanism. Microbiology and Molecular Biology Reviews, 70, 605-645. https://doi.org/10.1128/MMBR.00013-06

[14] Baroni, A., Perfetto, B., Paoletti, I., De Martino, L., Buommino, E., Ruocco, E. and Ruocco, V. (2001) Uptake of Malassezia furfur by Human Dermal Fibroblasts: Effect of Ketoconazole and Cytoskeleton Inhibitors. Archives of Dermatological Research, 293, 407-413. https://doi.org/10.1007/s004030100242

[15] Muñoz, D.A.R., Castrejón, J.N.S., Baltierra, U.S.L., Pérez, R.S.J., Carapia, M.N., Castañeda, S.J.I., Luna, H.J., López, S.R., Rodríguez, T.A.V. and García, P.B.E. (2016) Candida glabrata Survives and Replicates in Human Osteoblasts. Pathogens and Disease, 74, 1-11.

https://academic.oup.com/femspd/article/74/4/ftw030/2197983

[16] Ortega, G. (2016) Evaluation of the Changes Observed in the Actin Filaments of the RK13 Cell Line during Infection with Candida albicans. Thesis of Degree, National Autonomous University of Mexico, Ciudad de México.

[17] Baroni, A., Perfetto, B., Paoletti, I., Ruocco, E., Canozo, N., Orlando, M. and Buommino, E. (2001) Malassezia furfur Invasiveness in a Keratinocyte Cell Line (HaCat): Effects on Cytoskeleton and on Adhesion Molecule and Cytokine Expresion. Archives of Dermatological Research, 293, 414-419. https://doi.org/10.1007/s004030100248

[18] Martín, J.L., Tejedor, M.T., Lupiola, P., Morales, M. and González, Z. (2001) Relationship between the Presence of Malassezia pachydermatis and the Clinical Signs Found in Chronic Canine Otitis in a Population of Dogs of the Canarian Podenco Breed. Clínica Veterinaria Pequeños Animals, 21, 103-111. https://ddd.uab.cat/pub/clivetpeqani/11307064v21n2/11307064v21n2p103.pdf

[19] Zachary, J.F. and McGavin, M.D. (2012) Pathologic Basis of Veterinary Disease. 5th Edition, Elsevier, Amsterdam.

[20] Gupta, A., Kohli, Y. and Summerbell, R. (2000) Molecular Differentiation of Seven Malassezia Species. Journal of Clinical Microbiology, 38, 1869-1875. http://jcm.asm.org/content/38/5/1869.full.pdf

[21] Coutinho, S.D. and Paula, C.R. (2000) Proteinase, Phospholipase, Hyaluronidase and Chondroitin-Sulphatase Production by Malassezia pachydermatis. Medical Mycology, 38, 73-76. https://doi.org/10.1080/mmy.38.1.73.76

[22] Mauldin, E., Scott, D., Miller, W. and Smith, C. (1997) Malassezia Dermatitis in the Dog: A Retrospective Histopatological and Inmunopathological Study of 86 Cases (1990-1995). Veterinary Dermatology, 8, 191-202. https://doi.org/10.1046/j.1365-3164.1997.d01-15.x

[23] Cheng, T.A. and Hill, P.B. (2005) The Biology of Malassezia Organisms and Their Ability to Induce Immune Responses and Skin Disease. Veterinary Dermatology, 16, 4-26. https://doi.org/10.1111/j.1365-3164.2005.00424.x

[24] Mathieson, I., Fixter, L. and Little, C. (1998) Enzymatic Activity of Malassezia pachydermatis. In: Kwochka, K.W., Willemse, T. and von Tscharner, C.V., Eds., Advances in Veterinary Dermatology, Vol. 3, Butterworth Heinemann, Oxford, 532. 
[25] Kopecka, M., Yoshida, S. and Yamaguchi, M. (2012) Actin Ring Formation around the Cell Nucleus of Long-Neck Yeast. Journal of Electron Microscopy, 61, 249-255. https://doi.org/10.1093/jmicro/dfs049

[26] Kopecka, M., Kawamoto, S. and Yamaguchi, M. (2013) A New F-Actin Structure in Fungi: Actin Ring Formation around the Cell Nucleus of Cryptococcus neoformans. Microscopy, 62, 295-301. https://doi.org/10.1093/jmicro/dfs074 